\title{
GOLD \\ ELECTRODEPOSITION WITHIN THE ELECTRONICS INDUSTRY
}

\author{
Ian R. Christie \& Brian P. Cameron \\ GEC-Marconi Limited \\ Hirst Research Centre, Hertfordshire \\ England
}

The use of gold within the electronics industry is widespread for

domestic, commercial, aerospace and defence equipment.

The combination of good electrical conductivity coupled with high resistance to corrosion has led to its widespread adoption as the standard material for contacts, bonding, joining, and high performance high reliability conductor applications. In part due to its high intrinsic cost, gold is usually employed in the form of a thin layer and the performance of this relatively thin film is critical to the correct functioning of many devices. This article deals with the production of these thin gold layers by electrodeposition processes which play an important part in processing technology. 
The electrodeposition of gold is in itself not a new process, the first record of gold electroplating being in 1802 by Luigi $V$. Brugnatelli [1]. However, it was not until 1840 that H. and G.R. Elkington [2] patented the forerunner of modern gold electroplating formulations based on the double salt gold potassium cyanide. From 1845 the process was commercially available for decorative gold electroplate. However, the understanding of the process was poor and hence processing was somewhat unreliable, much of the success being dependent on operator skill. It remained relegated to the production of decorative items until its re-emergence as part of the electronics industry in the 1940s. This new industrial interest grew rapidly and electroplating became the major user of gold for the electronics industry. For example, in 1990 the total usage of gold for the European electronics industry was estimated as $18,097 \mathrm{~kg}$ of which $13,482 \mathrm{~kg}$ was electrodeposited [3]. For 1990, world consumption of gold within the electronics industry was $142,800 \mathrm{~kg}$ [4]. This would indicate that approximately $110,000 \mathrm{~kg}$ was electrodeposited.

\section{ELECTROPLATING FORMULATIONS}

Two major requirements for the electronics industry exist which are satisfied by electroplated gold. One is a high purity gold primarily used for bonding or joining operations or where highest electrical conductivity is at a premium and the second where a hard wear resistant coating is required in contact and connector applications. The chemistry behind the two processes, whilst both based on gold potassium cyanide, is somewhat different and requires some knowledge and understanding of electrodeposition processes.

\section{Pure Gold}

Soft pure gold needs to be electrodeposited from electrolytes containing the minimum of impurities and ideally should not contain any foreign element or organic material which could co-electrodeposit with the gold. Hence, a need for high purity electrolyte chemicals and electroplating conditions which do not favour co-electrodeposition. Most formulations consist of the gold salt, as the source of metal ions, a buffer material to ensure that the $\mathrm{pH}$ of the solution remains effectively constant over an extended time period, a $\mathrm{pH}$ adjuster and, in some cases, salts to increase electrolyte conductivity.

Typical formulations and operating conditions are given in Table 1. Note that to obtain high quality electrodeposits the plating cathode current density is kept low and hence deposition rates are low.

Replenishment of gold is carried out via the addition of gold as gold potassium cyanide. An inert anode is used, usually platinised titanium rather than soluble gold anodes. There are several reasons for this practice. The electrolyte is not $100 \%$ efficient with respect to gold deposition, whereas anodic dissolution of the metal is much closer to $100 \%$ and the use of soluble gold anodes would soon result in high electrolyte gold concentration and the balance of the solution would be adversely effected. Also the cost of gold anodes of any size would increase inventory costs. To ensure the production of consistently good quality deposits the electrolyte has to be regularly analyzed and deficiencies due to chemical consumption or drag out losses made good. Over an

Table 1

Pure soft gold electrolytes 
Table 2 Acid golds

extended time period most electrolytes build up decomposition products which have to be removed, usually by treatment with active charcoal. After such a treatment a full analysis of chemical composition is carried out followed by trials with test coupons.

\section{Hard Gold}

The second most important electrolyte used by the electronics industry is one designed to produce transition metal hardened deposits. Pure gold electroplated from neutral or alkaline electrolytes is often used - because of its softness - for its ability to thermocompression bond but this very property excludes such deposits for applications where contacts and connectors are employed. The pure gold deposit is capable of welding to itself when contact is made, resulting in a permanently closed contact.

To overcome this problem, transition metal hardened gold electrodeposits are employed, produced from electrolytes operating in the acid $\mathrm{pH}$ range. The presence of such metals as nickel, cobalt or iron in such electrolytes results in codeposition of a gold/transition metal alloy. Codeposited with the transition metal is organic material, probably deriving from cyanide polymerisation [5] which acts as a lubricant within the gold film. Inclusion of these materials considerably alters the properties of the deposit giving a significant rise in deposit hardness and wear resistance. The presence of these impurities reduces the tendency of the gold layer to weld by friction to an extent that makes this material very attractive for connectors, sliding contacts and contact applications, and it is widely used throughout the industry.
Bath composition $(\mathrm{g} / \mathrm{l})$ and operating conditions

I.

8

40

40

-

5

5

-

- $\quad-\quad 1$

$22-25$

$5-20$

40

20
3.

12

05

$-$

-

-

35

5

Some examples of formulations employed are given in Table 2 and the two most important differences in the formulations and operations of the electrolyte are the inclusion of a chelated metal complex of, usually, cobalt or nickel and the $\mathrm{pH}$ operation of the electrolyte, usually in the range 3.5 - 4.5. Despite the acid nature of the electrolyte, cyanide decomposition occurs only slowly but the bath is unlikely to retain any free cyanide as this will be evolved as the complex decomposes during electrodeposition of gold.

The composition of the deposit from these acid gold electrolytes contains up to approximately $0.7 \%$ of the transition metal. The complex co-ordinating ligand employed to complex the transition metal salt in solution is important as this controls the amount of free transition metal in the electrodeposit. If the complex is chemically too strong, low transition metal content alloys are deposited, with reduced wear resistance and hardness. If the complex is too weak, then high transition metal content deposits are produced which result in stress cracking and, in severe cases, exfoliation of the electrodeposit. Indeed, with these baths care has to be taken that no transition metals are allowed to contaminate the solution via drag-in or in the case of iron by chemical corrosive attack.

The prime applications for these acid hard gold electrolytes are within the connector industry. Hence, a mass produced item with selective deposition of gold 
requires the highest deposition rates to reduce process time to a minimum. This is usually achieved by rapid agitation, for example, by jetting the electrolyte onto the selectively masked substrate and hence, for these applications, high gold content electrolytes are employed. Typical examples are given in Table 2.

\section{Immersion Gold}

In many applications an immersion gold solution is employed to deposit a very thin (sub micron) layer onto the surface of electronic components. For example, transistor leads are often immersion gold coated to aid solderability of the wires; the gold retards the oxidation of the underlying nickel electroplate. In recent years, gold immersion deposits have become important in printed circuit applications where an immersion coating improves the performance of printed pressure contacts and acts as an etch resist.

The process relies on the ability of noble metals in solution to replace base metals, a simple example being the replacement of iron by copper when iron is immersed in aqueous copper sulphate solution. This displacement will occur depending on the value of the electrode potential of the metals concerned. Examples of electrode potentials are given in Table 3, but note that the electrode potential can be considerably altered by complexing the metal ion. Normally a metal in this series will replace any metal below it on the list. However, if the potential difference is too great, spongy non-adherent deposits are formed due to the speed of reaction being too great. Most of the immersion solutions available are proprietary but all contain gold as the double cyanide and are usually alkaline. However, one of the authors has demonstrated that acid golds can produce acceptable deposits on electroplated nickel with good adhesion and of bright appearance.

In general the deposits are very thin and afford only marginal protection from corrosion. Nonetheless, the rapidity of the process, usually complete within two minutes of immersion, coupled with the lack of requirements for precision electroplating and the improved appearance to the component, makes such deposits popular substitutes for thicker gold electrodeposits. They are not to be recommended for any high specification electronic components or service environments likely to encounter aggressive atmospheres. However, their main usage as a protective film during storage for subsequent hot dip soldering has proved beneficial.

\section{Autocatalytic or Electroless Gold}

The re-emergence of immersion gold as a production process has prompted new research into autocatalytic gold deposition as, for some applications, deposits of greater thickness than those available from gold immersion processes (where the layers are sub-micron in thickness) are required. For example in the processing of complex waveguide structures the use of a uniform relatively thick (two micron) gold layer over the surface, including recesses, would be advantageous. The gold deposition occurs not by the passage of a current but by the action of a reducing agent.

The solutions are carefully formulated so that the gold deposition only occurs at the metal surface to be plated and do not lead to the formation of free gold in the bulk solution. Solutions of this type are widely used for the deposition of copper and nickel and the same principles are now being applied to gold deposition.

\section{PROPERTIES OF GOLD ELECTRODEPOSITS}

One unique feature of all electrodeposited coatings including gold is the extremely fine grained nature of the electrodeposited film. All thermally produced metals in bulk exhibit larger grain sizes. This means that the metallurgical properties of electrodeposits 


\begin{tabular}{|l|c|c|c|c|c|}
\multicolumn{1}{c}{$\begin{array}{c}\text { Table } 4 \\
\text { Properties of gold electrodeposits }\end{array}$} & $\begin{array}{c}\text { Gold } \\
\text { content } \\
\%\end{array}$ & $\begin{array}{c}\text { Hardness } \\
(\mathbf{K V} \text { N25) }\end{array}$ & $\begin{array}{c}\text { Resistivity } \\
(\mu \Omega . \mathbf{c m})\end{array}$ & $\begin{array}{c}\text { Contact } \\
\text { resistance } \\
(\mathbf{m} \Omega)\end{array}$ \\
\hline & Pure gold deposit & 100 & $40-90$ & 2.4 & 0.3 \\
& Cobalt gold & $99.5+$ & $120-250$ & 15.0 & 0.6 \\
& Nickel gold & $99.3+$ & $160-200$ & 11.0 & 0.3 \\
\hline
\end{tabular}
of gold and gold alloys.

This in turn gives these electrodeposited coatings applications not normally expected for gold metal.

Table 4 illustrates values obtained on hardness and electrical properties of pure gold and alloyed hard golds. As can be seen, the presence of trace quantities of foreign metal considerably increases electrical resistivity but the effect on contact resistance is considerably less. The latter measurement is in part a measure of the surface cleanliness of the contacting surfaces and also a measure of the applied force.

Grain size of all the electrodeposited golds is extremely fine being sub-micron and difficult to resolve by optical methods. This is in stark contrast to gold and gold alloys metallurgically prepared via conventional routes and is reflected by the relatively high hardness values for electrodeposited gold. In the case of the cobalt and nickel gold alloys, the presence of entrapped organic material codeposited with the gold via cyanide polymerisation can give a layer-type structure with the polymeric material deposited as a discontinuous film through the structure. This, in effect, reduces the specific gravity of the deposit.

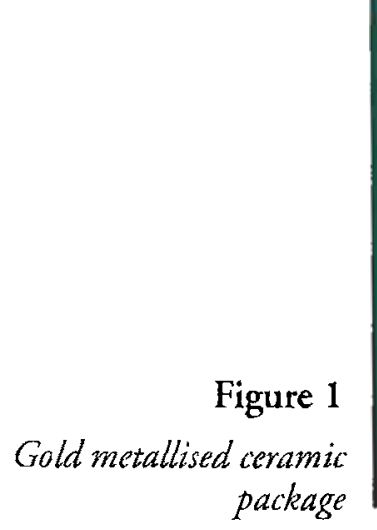

\section{APPLICATIONS}

In the electronics industry gold is electrodeposited in order to make use of its excellent characteristics in terms of its electrical, chemical and optical properties. It is convenient to consider the applications utilising this unique combination of characteristics according to the type of deposit/process.

\section{Pure Gold}

Pure gold is often referred to as soft gold because of its low hardness (approximately 40-60 vhn). Considerable use is made of its relative softness for bonding. A compression bond can be made simply by bringing together two gold plated surfaces (or commonly a gold wire and a gold plated surface) and

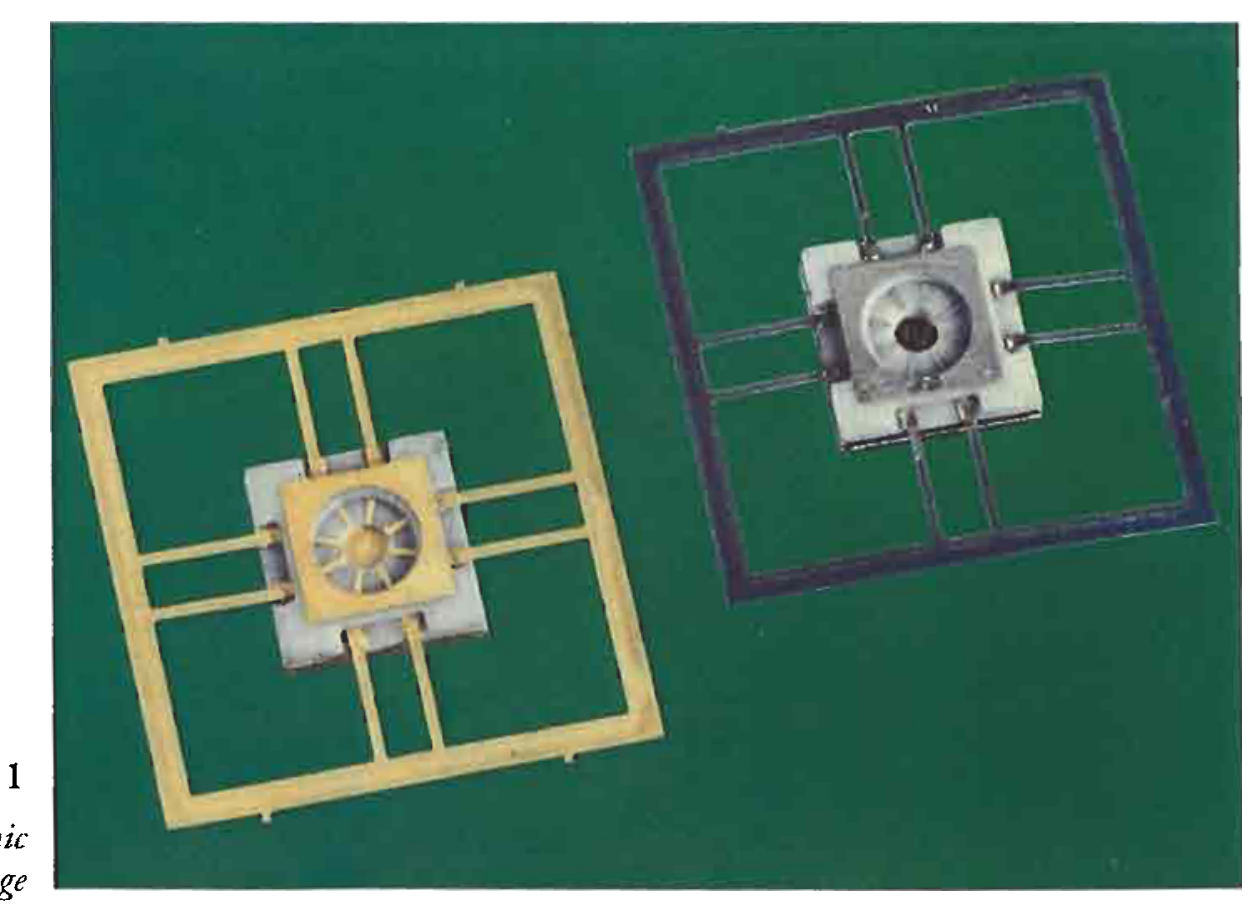


applying pressure. The absence of an oxide layer on the surface coupled with high diffusion rates results in a rapid joining process. Additionally, heat may be applied.

Use is made of compression bonding or thermal compression bonding to make connections in semiconductor devices, to seal packages such as semi-conductor cans and in a variety of joining applications. This process is carried out on a wide variety of silicon devices to make a connection between the chip and the substrate leads (Fig. 1). In many cases the device is subsequently packed in a hermetically sealed can, again using the thermo-compression bonding process to seal the can. The device itself may have a large number of connections berween the chip and the external unit, as illustrated in Figure 2. In long production runs there may be millions of joints to be processed requiring a high degree of reliability and consistency in operation.

An alternative process, known as TAB (for tape automated bonding) removes the need for a gold wire between the silicon chip and the lead frame. The lead frame is selectively plated to apply gold only to those locations to be bonded. The components are in the form of a tape or strip to allow a continuous process. The chip itself is also selectively plated. By compression bonding of the lead frame to the chip, good joints are made with the need for only one connection rather than the two required for wire bonding connections.

Pure gold is widely used in the fabrication of microwave integrated circuits for communication, aero-

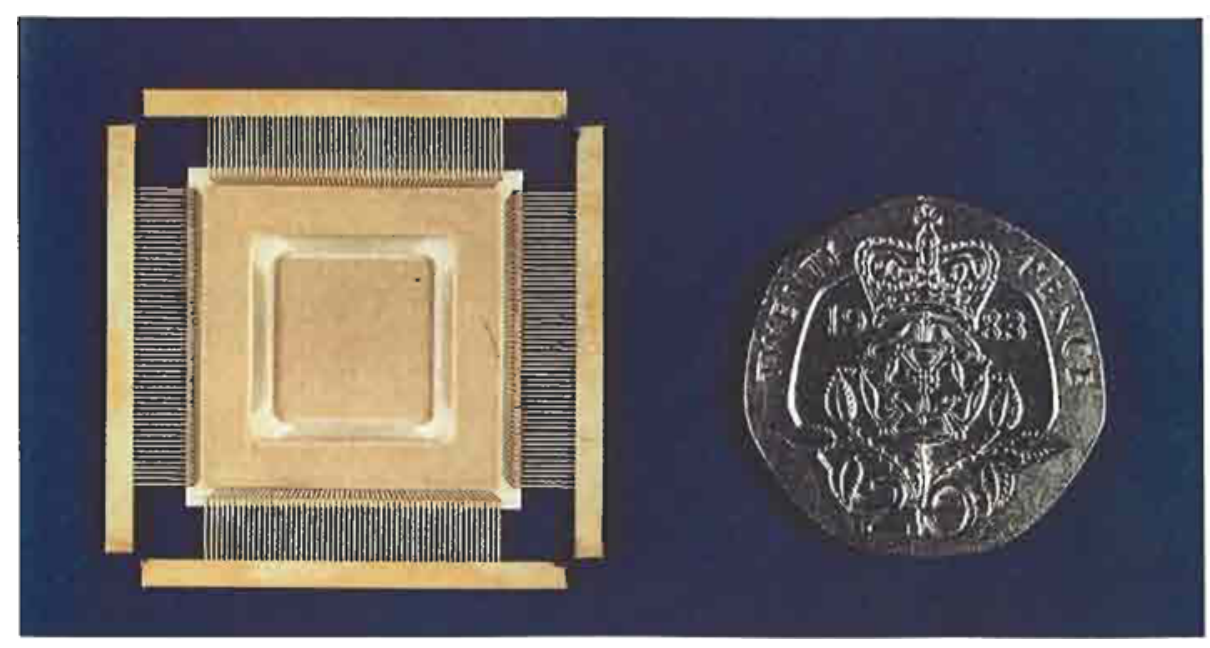

space and military applications. Here the dielectric properties of conventional printed circuit laminate are inadequate and use is made of high purity alumina or quartz as the substrate material. The substrate is first metallised by vacuum deposition of a nickel-chromium alloy followed by a thin $(2000 \AA)$ layer of gold. Conductivity of the gold layer is then enhanced to the required level by the electrodeposition of pure gold to thicknesses of up to twenty-five microns. Subsequent masking and etching produce a suitable pattern of tracks and bonding pads on the substrate [6], examples are shown in Figures 3,4 and 5. An alternative is to vacuum metallise and electroplate only those areas required.

Plated gold layers also provide readily solderable surfaces. Here again the absence of an oxide layer contributes to the ease of processing. As gold can form brittle inter-metallics with tin when the gold content exceeds approximately $4 \%$, the amount of gold going into, say, a $\mathrm{Pb}-\mathrm{Sn}$ joint must be carefully controlled or alternatives, such as indium based solders, used.

\section{Hard Gold}

As discussed previously, hard gold alloys are employed particularly in those sections of the industry where the contact could be subjected to wear by make and break or by insertion and withdrawal. The unique combination of properties combining high corrosion resistance with good lubrication (and hence low wear rates) has made hard golds the main contender for the top coat of contacts. Alternatives, such as palladium and palladium-nickel, have been proposed but gold is by far the most widely employed material.

Figure 2

Gold electroplated lead form bonded to metallised chip (note the large number of interconnects) 


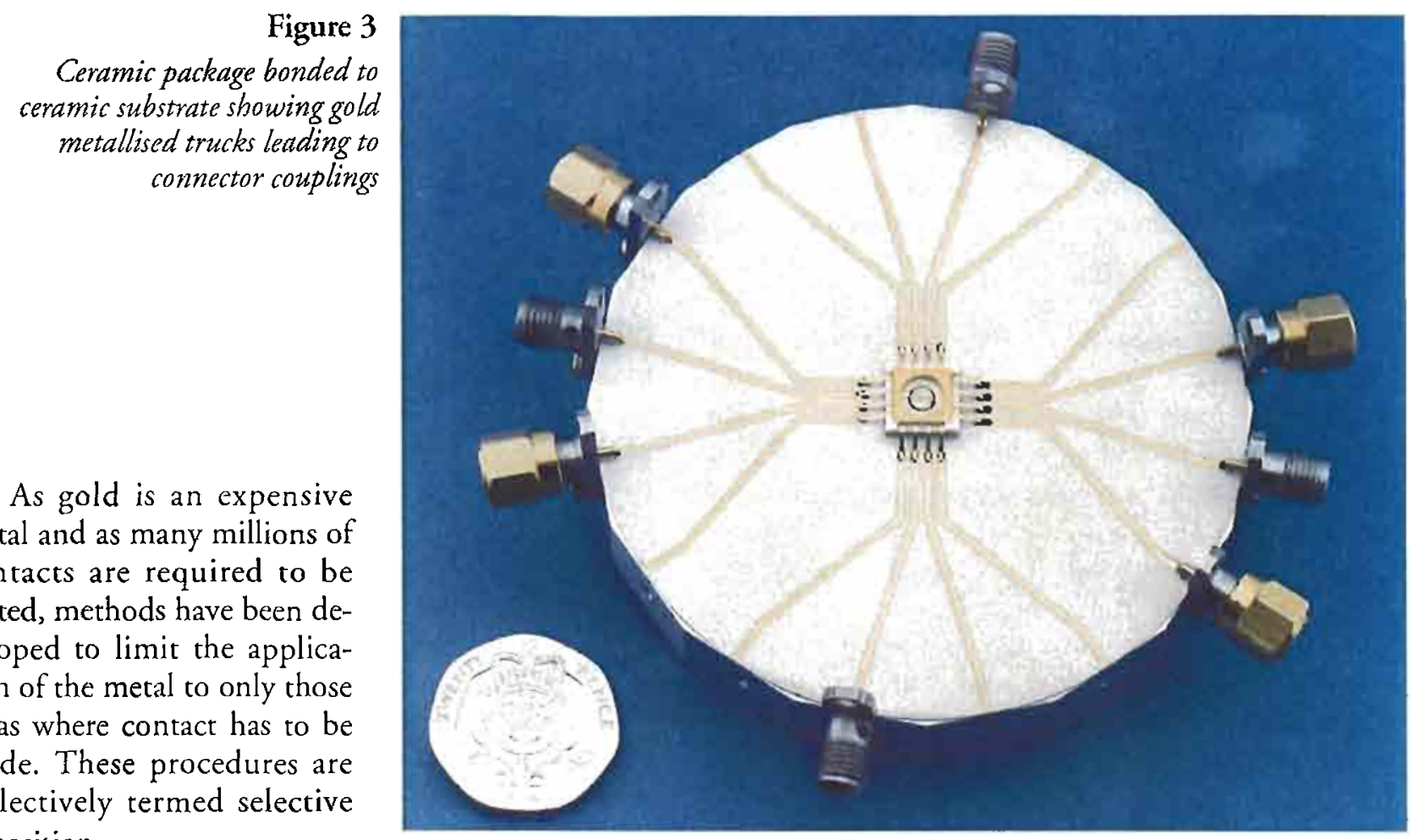

deposition.

The simplest of these is to only partly immerse the connector in the gold electrolyte, thus reducing the area plated. However, as the thickness of the gold layer can be relatively high (typically 0.8 to $2.5 \mu \mathrm{m}$ ) excess metal is still deposited as (a) both sides of the connector are plated whereas often contact is only made on one side and (b) it is difficult to control the exact immersion depth, leading to difficulties in controlling deposit thickness.

A more satisfactory method is to mask both sides of the connector, one side fully and the orher so as to allow solution access only to the area which requires plating. This type of process is capable of great precision with respect to gold placement as well as thickness control but is more expensive in terms of plant and the variety of masks required to accept a wide range of connector types.

Most connectors are electroplated using a reel-toreel process where the connectors are stamped from the base metal, often phosphor bronze, in the form of a continuous strip. The strip is subsequently processed through a series of cleaning, etching and electroplating baths as a continuous reel. This process enables large numbers of connectors to be processed in large volumes with minimal labour costs (Fig.6).
Gold electrodeposits can be plated which contain small (up to $0.7 \%$ ) amounts of nickel, cobalt or iron. This alloying addition increases the hardness of the deposit to the region of 80-200 vhn.

This type of gold deposit is more suitable for contact applications. Soft gold deposits would quickly be smeared and deformed by insertion forces. Gold alloy deposits do not just depend on their increased hardness for their resistance to wear. A small amount of co-polymer material from the complexes present in the gold plating solution is also incorporated into the deposit and this improves the wear resistance by a minute degree of lubrication. With a contact resistance of the order of a few milli-ohms hard gold deposits form the ideal contact material. Telephone exchanges are just one example of the high-volume applications of gold plated connectors where good corrosion and wear resistance combined with a low contact resistance play a vital role.

An additional benefit comes from the fact that gold does not catalyse polymeric reactions. Palladium or palladium alloy finishes can cause polymeric material in the atmosphere to be polymerised to form a surface film which increases the contact resistance. 


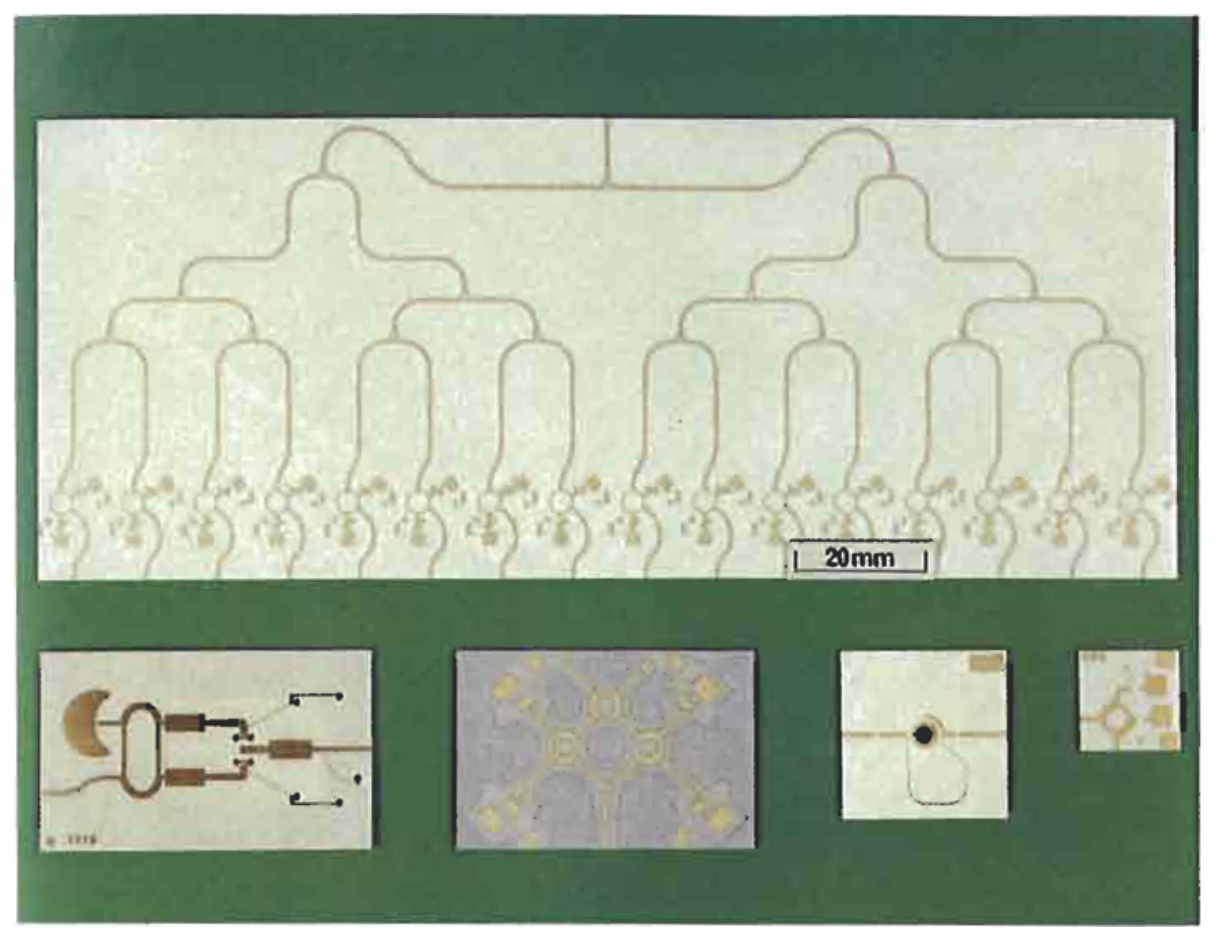

Figure 4

Gold metallised

ceramic microwave tracks

considerable engineering problems of mask alignment, development is currently under way on developing new rechniques which direct a high velocity jet of electrolyte at the surface; under the correct deposition conditions gold is only plated where the electrolyte impinges on the surface.

As with many other applications for plated gold, considerable effort has been directed at the selective deposition of gold to minimise the amount of material used and thus the cost. This selective deposition can take the form of formulating solutions to give an improved distribution of metal or selective plating by the use of masks to prevent deposition. Because of the

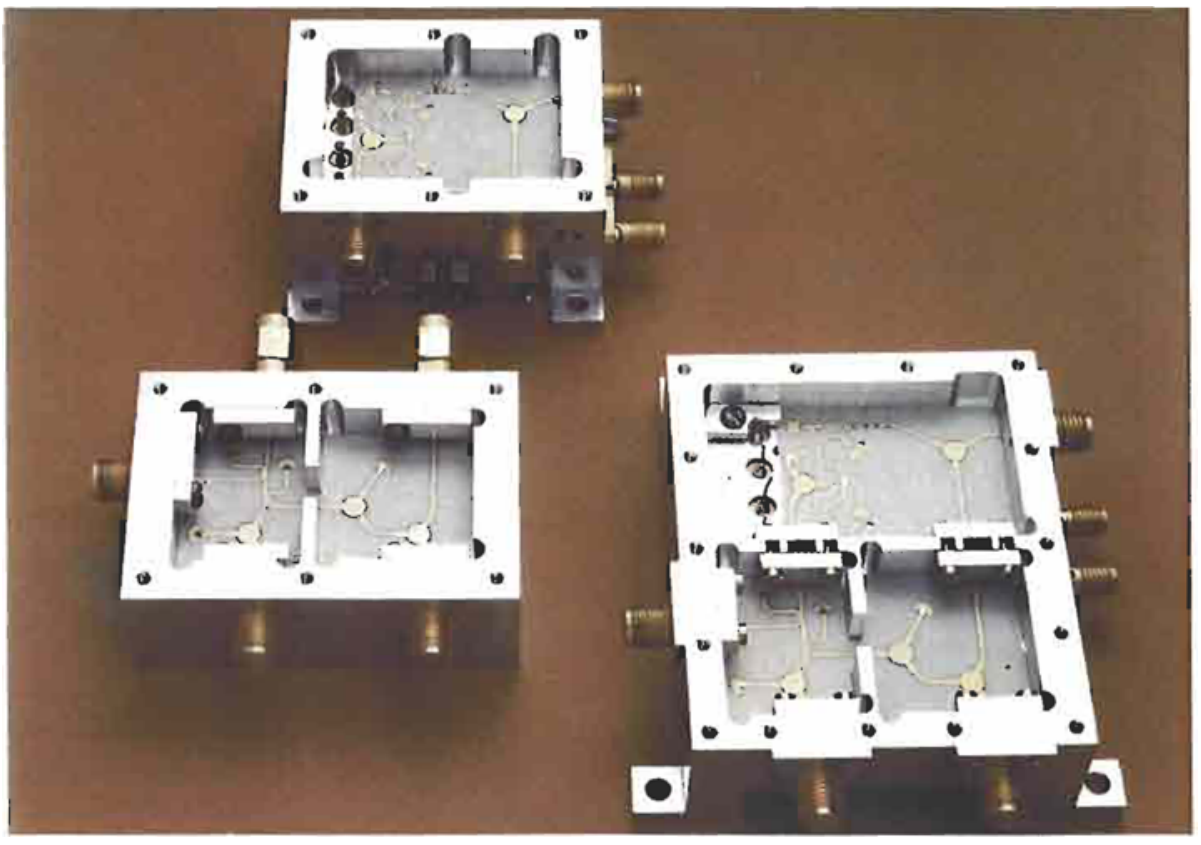

\section{Autocatalytic and Immersion Gold}

Autocatalytic and immersion golds are used where relatively thin films of gold are required. If components are isolated it is not possible to make electrical contact with individual areas and hence the part cannot be electroplated. Also if any fine line geometries are utilised the resistance of the line makes electrodeposition at best a process yielding wide variations in metal deposit thickness. An advantage of the autocatalytically deposited gold is that the deposits are uniform in thickness and thus it is possible to evenly coat very complex geometries.

Figure 5

Experimental J-band modules 
Figure 6

Gold electroplated contact zone in strip of reel-to-reel connector fermales

Applications for immersion gold are becoming more important as surface mount technologies within the industry slowly mature. By using a top coat of immersion gold over copper or nickel printed circuit tracks it is feasible to retain improved solderability for subsequent soldering operations. The gold retards or prevents oxidation of the substrate, presenting a good surface for subsequent operations. As the process is a simple immersion technique it is gathering in importance throughout the industry.

\section{CONCLUSION}

Electroplated gold continues to play an important part in modern electronic technology. It is difficult to see a truly equivalent substitute due to the unique combination of properties of the metal. As the communication and information technologies continue to expand it is to be expected that the quantity of gold used by the industry will continue to increase.

\section{REFERENCES}

1. L.V. Brugnatelli, Ann. Chim. (Pavia), 1802, 21, 148

2. G.R. Elkington, British patent 8447,1840

3. 'Gold 1991', Goldfield Mineral Services Ltd., London 1991

4. 'The Consumption of Gold Products by Western European Electronics and Electrical Industries', 3rd Quarter and whole year, G.G. Wedgewood Services, Dec. 1991

5. G.B. Munier, Plating and Surface Finishing, 1960, 56,1159

6. 'Electroplated Gold in Microwave Integrated Circuits', I.R. Christie \& W. Mazur, Gold Bull., $1986,19(2)$ 